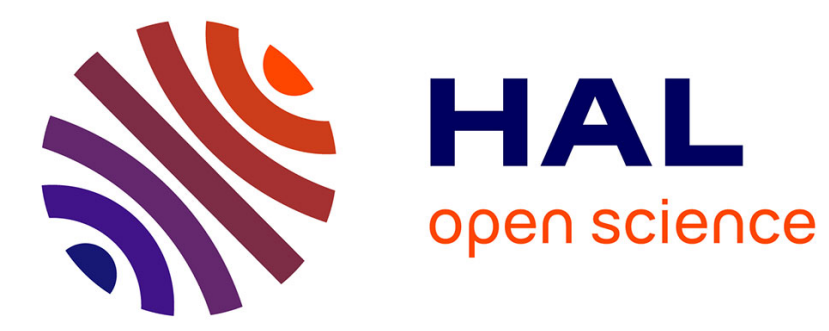

\title{
Probabilistic analysis of strip footings resting on a spatially random soil using subset simulation approach
}

Ashraf Abd El-Modby Soliman Ahmed, Abdul-Hamid Soubra

\section{To cite this version:}

Ashraf Abd El-Modby Soliman Ahmed, Abdul-Hamid Soubra. Probabilistic analysis of strip footings resting on a spatially random soil using subset simulation approach. Georisk: Assessment and Management of Risk for Engineered Systems and Geohazards, 2012, 6 (3), pp.188-201. 10.1080/17499518.2012.678775 . hal-01007213

\section{HAL Id: hal-01007213 \\ https://hal.science/hal-01007213}

Submitted on 11 Mar 2018

HAL is a multi-disciplinary open access archive for the deposit and dissemination of scientific research documents, whether they are published or not. The documents may come from teaching and research institutions in France or abroad, or from public or private research centers.
L'archive ouverte pluridisciplinaire HAL, est destinée au dépôt et à la diffusion de documents scientifiques de niveau recherche, publiés ou non, émanant des établissements d'enseignement et de recherche français ou étrangers, des laboratoires publics ou privés. 


\title{
Probabilistic analysis of strip footings resting on a spatially random soil using subset simulation approach
}

\author{
Ashraf Ahmed and Abdul-Hamid Soubra* \\ Department of Civil Engineering, University of Nantes, Saint-Nazaire Cedex, France
}

\begin{abstract}
The failure probability of geotechnical structures with spatially varying soil properties is generally computed using Monte Carlo simulation (MCS) methodology. This approach is well known to be very time-consuming when dealing with small failure probabilities. One alternative to MCS is the subset simulation approach. This approach was mainly used in the literature in cases where the uncertain parameters are modelled by random variables. In this article, it is employed in the case where the uncertain parameters are modelled by random fields. This is illustrated through the probabilistic analysis at the serviceability limit state (SLS) of a strip footing resting on a soil with a spatially varying Young's modulus. The probabilistic numerical results have shown that the probability of exceeding a tolerable vertical displacement $\left(P_{\mathrm{e}}\right)$ calculated by subset simulation is very close to that computed by MCS methodology but with a significant reduction in the number of realisations. A parametric study to investigate the effect of the soil variability (coefficient of variation and the horizontal and vertical autocorrelation lengths of the Young's modulus) on $P_{\mathrm{e}}$ was presented and discussed. Finally, a reliability-based design of strip footings was presented. It allows one to obtain the probabilistic footing breadth for a given soil variability.
\end{abstract}

Keywords: subset simulation; Monte Carlo simulation; random field; spatial variability; Karhunen-Loeve expansion

\section{Introduction}

During the recent years, much effort has been paid for the probabilistic analysis of geotechnical structures. Some simplified methods have modelled the different uncertain parameters by random variables where the soil is considered as a uniform material. However, in nature, the soil parameters (shear strength parameters, elastic properties, etc.) vary spatially in both the horizontal and the vertical directions as a result of depositional and postdepositional processes. This leads to the necessity of representing the soil parameters as random fields characterised not only by their marginal probability density functions (as is the case of random variables), but also by their autocorrelation functions. In this regard, more advanced probabilistic approaches were proposed in the literature. These approaches are generally based on the finite element or the finite difference method. In these approaches, one needs to discretise the random field into a finite number of random variables. Once the random field is discretised into a finite number of random variables, the failure probability can be determined. In the framework of these approaches, Monte
Carlo simulation (MCS) is generally used to perform the probabilistic analyses.

Notice that MCS methodology is not suitable for the computation of a small failure probability because the number of simulations required becomes very large in this case. Au and Beck (2001) proposed an efficient approach (called subset simulation) to calculate the small failure probabilities in case where the uncertain parameters are modelled by random variables. In this approach, the failure probability is expressed as a product of conditional probabilities of some chosen intermediate failure events. Thus, the problem of evaluating a small failure probability in the original probability space is replaced by a sequence of events in the conditional probability space. Except Au et al. (2010) and Santoso et al. (2010) who applied the subset simulation approach to one-dimensional (1D) random field problems, the subset simulation method was mainly applied in the literature to problems where the uncertain parameters were modelled by random variables.

In this article, the subset simulation method was employed to perform a probabilistic analysis at the serviceability limit state (SLS) of a rigid strip footing resting on a soil with a two-dimensional (2D) spatially

*Corresponding author. Email: Abed.Soubra@univ-nantes.fr 
varying Young's modulus. The footing is subjected to a central vertical load (P). Notice that most previous studies that considered the soil spatial variability have modelled the uncertain parameters by isotropic random fields (e.g. Fenton and Griffiths 2003, Popescu et al. 2005, Griffiths et al. 2006, and Babu et al. 2006 for the ultimate limit state (ULS) analysis and Fenton and Griffiths 2002, 2005, Fenton et al. 2003 for the SLS analysis). However, due to the layered nature of soils, their parameters generally exhibit a larger autocorrelation length in the horizontal direction compared to that in the vertical direction. Thus, the Young's modulus is considered in this article as an anisotropic random field. The Karhunen-Loeve (K-L) expansion is used to discretise the random field. The deterministic model employed for the computation of the system response is based on numerical simulations using the commercial software FLAC. It should be emphasised here that the soil spatial variability causes uneven footing displacement. Due to its high rigidity, the footing undergoes a linear vertical displacement. Thus, the average value of the footing displacement is considered herein to represent the system response. This average is equal to the displacement at the footing centre.

After a brief description of the K-L expansion and the subset simulation method, the implementation of the subset simulation approach in the case of a random field problem is presented. Then, the probabilistic analysis of a strip footing resting on a spatially varying soil and the corresponding results are presented and discussed. This article ends with a conclusion.

\section{Karhunen-Loeve expansion}

In this article, the Young's modulus $E$ is modelled by a random field $E(X, \theta)$ where $X$ denotes the spatial coordinates and $\theta$ indicates the random nature of the Young's modulus. If $\mu$ is the mean of the Young's modulus, then the random field can be approximated by the K-L expansion as follows (Spanos and Ghanem 1989):

$$
E(X, \theta) \approx \mu+\sum_{i=1}^{M} \sqrt{\lambda_{i}} \phi_{i}(X) \xi_{i}(\theta)
$$

where $M$ is the size of the series expansion, $\lambda_{i}$ and $\phi_{i}$ are the eigenvalues and eigenfunctions of the covariance function $C\left(X_{1}, X_{2}\right)$, and $\xi_{i}(\theta)$ is a vector of standard uncorrelated random variables. The choice of the number of terms $M$ of the K-L expansion depends on the desired accuracy of the problem being treated. The error estimate $\operatorname{crr}(X)$ of the K-L expansion with $M$ terms can be calculated as follows (Sudret and Beveiller 2008):

$$
\operatorname{crr}(X)=1-(1 / \sigma) \sum_{i=1}^{M} \lambda_{i} \phi_{i}^{2}(X)
$$

The random field $E$ was assumed to follow a lognormal probability density function so that $\ln (E)$ is a normal random field with mean value $\mu_{\mathrm{ln}}$ and standard deviation $\sigma_{\ln }$. The choice of a log-normal probability density function is motivated by the fact that the Young's modulus is strictly non-negative (Fenton and Griffiths 2002). For a lognormal random field, Equation (1) becomes (Cho 2010, Cho and Park 2010):

$$
E(X, \theta) \approx \exp \left[\mu_{\mathrm{ln}}+\sum_{i=1}^{M} \sqrt{\lambda_{i}} \phi_{i}(X) \xi_{i}(\theta)\right]
$$

On the other hand, it was assumed that the Young's modulus follows an exponential covariance function. In the case of a log-normal random field, the exponential covariance function is given by:

$\left.C\left[\left(x_{1}, y_{1}\right),\left(x_{2}, y_{2}\right)\right]=\sigma_{\text {In }}^{2} \exp -\frac{\left|x_{1}-x_{2}\right|}{l_{\text {In } x}}-\frac{\left|y_{1}-y_{2}\right|}{l_{\text {In } y}}\right)$

where $\left(x_{1}, y_{1}\right)$ and $\left(x_{2}, y_{2}\right)$ are the coordinates of two arbitrary points in the domain $D$ over which the random field is defined, and $l_{\ln x}$ and $l_{\ln y}$ are respectively the horizontal and vertical lengths over which the values of log-elastic modulus are highly correlated. Notice that in the case of an exponential covariance function, the eigenvalues and eigenfunctions are given analytically. Their solutions are presented in Spanos and Ghanem (1989).

\section{Subset simulation}

Subset simulation was proposed by $\mathrm{Au}$ and Beck (2001) to compute the small failure probabilities in the case where the different uncertain parameters are modelled by random variables. This method was found to be an efficient tool for the computation of small failure probabilities compared to the crude MCS methodology. The basic idea of subset simulation is that the small failure probability can be expressed as a product of larger conditional failure probabilities. This is explained in more details in the following subsection.

\section{Basic idea of subset simulation}

Consider a failure region $F$ defined by the condition $G<0$ where $G$ is the performance function and let 
$\left(s_{1}, \ldots, s_{k}, \ldots, s_{N_{N}}\right)$ be $N_{t}$ samples located in the space of the uncertain variables where ' $s$ ' is a vector of random variables. It is possible to define a sequence of nested failure regions $F_{1}, \ldots, F_{j}, \ldots, F_{m}$ of decreasing size where $F_{1} \supset \ldots \supset F_{j} \supset \ldots \supset F_{m}=F$ (Figure 1). An intermediate failure region $F_{j}$ can be defined by $G_{j}<C_{j}$ where $C_{j}>0$. Thus, there is a decreasing sequence of positive numbers $C_{1}, \ldots, C_{j}, \ldots, C_{m}$ corresponding respectively to $F_{1}, \ldots, F_{j}, \ldots, F_{\mathrm{m}}$ where $C_{1}>\ldots>C_{j}>\ldots>C_{m}=0$. The $N_{t}$ samples $\left(s_{1}, \ldots\right.$, $\left.s_{k}, \ldots, s_{N_{t}}\right)$ will be divided into groups with equal number $N_{s}$ of samples $\left(s_{1}, \ldots, s_{k}, \ldots, s_{N_{s}}\right)$. Thus, $N_{t}=m \times N_{s}$ where $m$ is the number of failure regions. The first $N_{s}$ samples are generated according to MCS methodology following a target PDF $\left(\mathrm{P}_{\mathrm{t}}\right)$. The next $N_{s}$ samples of the different subsequent failure regions are obtained using Markov chain method based on Metropolis-Hastings (M-H) algorithm according to a proposal PDF $\left(\mathrm{P}_{\mathrm{p}}\right)$ as will be explained in the next subsection. The conditional failure probability corresponding to an intermediate failure region $F_{j}$ is calculated as follows:

$$
P\left(F_{j} \mid F_{j-1}\right)=\frac{1}{N_{s}} \sum_{k=1}^{N s} I_{F_{j}}\left(s_{k}\right)
$$

where $I_{F_{j}}=1$ if $s \in F_{j}$ and $I_{F_{j}}=0$ otherwise. The failure probability $P(F)=P\left(F_{\mathrm{m}}\right)$ of the failure region $F$ can be calculated from the sequence of conditional failure probabilities as follows:

$$
\begin{aligned}
P(F)= & P\left(F_{m}\right)=P\left(F_{m} \mid F_{m-1}\right) \times P\left(F_{m-1} \mid F_{m-2}\right) \times \\
& P\left(F_{m-2} \mid F_{m-3}\right) \times \ldots \times P\left(F_{2} \mid F_{1}\right) \times P\left(F_{1}\right)
\end{aligned}
$$

This equation can be regarded as a system consisting of $m$ components (related to the $\mathrm{m}$ failure regions

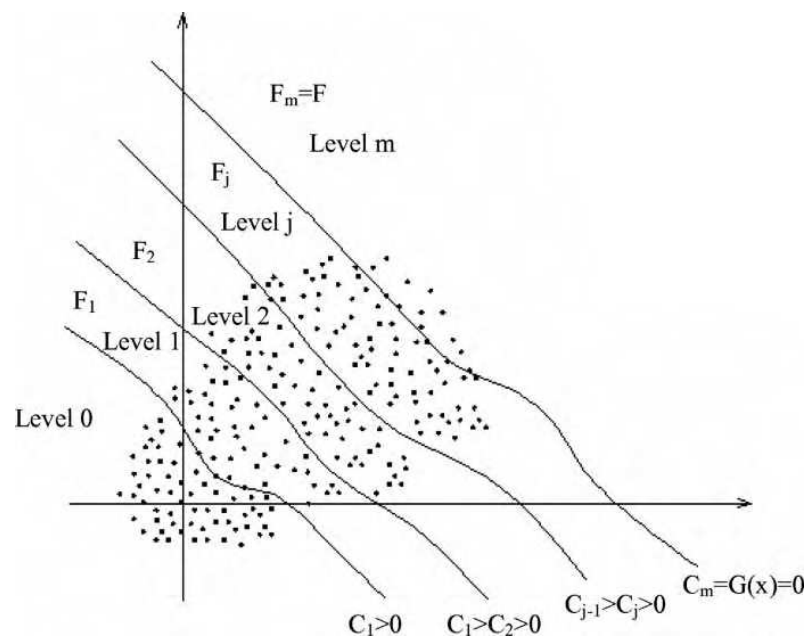

Figure 1. Nested failure domain.
$\left.F_{1}, \ldots, F_{j}, \ldots, F_{m}\right)$ connected in parallel. Consequently, the failure probability of the failure region $F$ is the intersection of all conditional failure probabilities of the failure regions $F_{1}, \ldots, F_{j}, \ldots, F_{m}$. Thus, the failure probability $P(F)$ is:

$$
P(F)=P\left(\cap_{j=1}^{m} F_{\mathrm{j}}\right)
$$

where

$$
\begin{aligned}
P\left(\cap_{j=1}^{m} F_{j}\right) & =P\left(F_{m} \mid \cap_{j=1}^{m-1} F_{j}\right) \times P\left(\cap_{j=1}^{m-1} F_{j}\right) \\
& =P\left(F_{m} \mid F_{m-1}\right) \times P\left(\cap_{j=1}^{m-1} F_{j}\right)=\ldots \\
& =P\left(F_{1}\right) \prod_{j=2}^{m} P\left(F_{j} \mid F_{j-1}\right)
\end{aligned}
$$

\section{Metropolis-Hastings algorithm}

The Metropolis-Hastings algorithm is a Markov chain Monte Carlo (MCMC) method. It is used to generate a sequence of random samples from existing samples (that were generated with a target PDF called ' $\mathrm{P}_{\mathrm{t}}$ ') by using a proposal PDF called ' $\mathrm{P}_{\mathrm{p}}$ '. Let $s_{k} \in F_{j}$ be a current sample which follows a target PDF ' $\mathrm{P}_{\mathrm{t}}$ '. Using the proposal PDF ' $\mathrm{P}_{\mathrm{p}}$ ', a next sample $s_{k+1} \in F_{j}$ that follows the target PDF ' $\mathrm{P}_{\mathrm{t}}$ ' can be simulated from the current sample $s_{k}$ as follows:

Step a: a candidate sample $\hat{s}$ is generated using the proposal $\operatorname{PDF}\left(\mathrm{P}_{\mathrm{p}}\right)$. The candidate sample $\hat{s}$ is centered at the current sample $s_{k}$.

Step b: using the deterministic model, evaluate the value of the performance function $G(\hat{s})$ corresponding to the candidate sample $\hat{s}$. If $G(\hat{s})<C_{j}$ (i.e. $\hat{s}$ is located in the failure region $F_{j}$ ), set $s_{k+1}=\hat{s}$; otherwise, reject $\hat{s}$ and set $s_{k+1}=s_{k}$ (i.e. the current sample $s_{k}$ is repeated).

Step c: if $G(\hat{s})<C_{j}$ in the preceding step, calculate the ratio $r_{1}=\mathrm{P}_{\mathrm{t}}(\hat{s}) / \mathrm{P}_{\mathrm{t}}\left(s_{k}\right)$ and the ratio $r_{2}=\mathrm{P}_{\mathrm{p}}\left(s_{k} \mid \hat{s}\right) /$ $\mathrm{P}_{\mathrm{p}}\left(\hat{s} \mid s_{k}\right)$, then compute the value $r=r_{1} r_{2}$.

Step d: if $r \geq 1$ (i.e. $\hat{s}$ is distributed according to the $\mathrm{P}_{\mathrm{t}}$ ), one continues to retain the sample $s_{k+1}$ obtained in step b; otherwise, reject $\hat{s}$ and set $s_{k+1}=s_{k}$ (i.e. the current sample $s_{k}$ is repeated).

Notice that in step $\mathrm{b}$, if the candidate sample $\hat{s}$ does not satisfy the condition $G(\hat{s})<C_{j}$, it is rejected and the current sample $s_{k}$ is repeated. Also in step d, if the candidate sample $\hat{s}$ does not satisfy the condition $r \geq 1$ (i.e. $\hat{s}$ is not distributed according to the $\mathrm{P}_{\mathrm{t}}$ ), it is rejected and the current sample $s_{k}$ is repeated. The presence of several repeated samples is not desired as it leads to high probability that the chain of samples remains in the current state. This means that there is high probability that the next failure 
threshold $C_{j+1}$ is equal to the current failure threshold $C_{j}$ which decreases the efficiency of the subset simulation approach. To overcome this inconvenience, Santoso et al. (2010) have proposed to modify the classical M-H algorithm as follows:

Step a: a candidate sample $\hat{s}$ is generated using the proposal PDF $\left(\mathrm{P}_{\mathrm{p}}\right)$. The candidate sample $\hat{s}$ is centered at the current sample $s_{k}$.

Step b: calculate the ratio $r_{1}=\mathrm{P}_{\mathrm{t}}(\hat{s}) / \mathrm{P}_{\mathrm{t}}\left(s_{k}\right)$ and the ratio $r_{2}=\mathrm{P}_{\mathrm{p}}\left(s_{k} \mid \hat{s}\right) / \mathrm{P}_{\mathrm{p}}\left(\hat{s} \mid s_{k}\right)$, then compute the value $r=r_{1} r_{2}$

Step c: if $r \geq 1$, set $s_{k+1}=\hat{s}$; otherwise, another candidate sample is generated. Candidate samples are generated randomly until the condition $r \geq 1$ is satisfied.

Step d: using the deterministic model, evaluate the value of the performance function $G\left(s_{k+1}\right)$ of the candidate sample that satisfies the condition $r \geq 1$. If $G\left(s_{k+1}\right)<C_{j}$ (i.e. $s_{k+1}$ is located in the failure region $F_{j}$ ), one continues to retain the sample $s_{k+1}$ obtained in step c; otherwise, reject $\hat{s}$ and set $s_{k+1}=s_{k}$ (i.e. the current sample $s_{k}$ is repeated).

These modifications reduce the repeated samples and allow one to avoid the computation of the system response of the rejected samples. This becomes important when the time cost for the computation of the system response is expensive (i.e. for the finite element or finite difference models).

Finally, it should be mentioned that this modified $\mathrm{M}-\mathrm{H}$ algorithm is employed in this article to increase the efficiency of the subset simulation approach.

\section{Implementation of subset simulation approach in case of a spatially varying soil property}

As mentioned previously, this article aims at employing the subset simulation methodology for the computation of the failure probability in the case of a spatially varying soil property. In order to achieve this purpose, a link between the subset simulation approach and the K-L expansion was performed. It should be emphasised here that the K-L expansion includes two types of parameters (deterministic and stochastic). The deterministic parameters are the eigenvalues and eigenfunctions of the covariance function. The role of these parameters is to ensure the correlation between the values of the random field at the different points in the space. However, the stochastic parameters are represented by the vector of the standard normal random variables $\left\{\xi_{i}\right\}_{i=1, \ldots, M}$. The role of these parameters is to ensure the random nature of the uncertain parameter. The link between the subset simulation approach and the K-L expansion was performed through the vector $\left\{\xi_{i}\right\}_{i=1, \ldots, M}$.
This ensures that the subset simulation technique does not affect the correlation structure of the random field.

The basic idea of the link is that for a given random field realisation obtained by the K-L expansion, the vector $\left\{\xi_{i}\right\}_{i=1, \ldots, M}$ represents a sample 's' of the subset simulation method for which the system response is calculated in two steps. The first step is to substitute the vector $\left\{\xi_{i}\right\}_{i=1, \ldots, M}$ in Equation (3) to calculate the value of the random field at each point in the space according to its coordinates. The second step is to use the deterministic model to calculate the corresponding system response. The algorithm of subset simulation in case of a spatially varying soil property can thus be described as follows:

Step 1: choose the number $M$ of terms of the K-L expansion. This number must be sufficient to accurately represent the target random field.

Step 2: generate a vector of standard normal random variables $\left\{\xi_{1}, \ldots, \xi_{i}, \ldots, \xi_{M}\right\}$ by direct MCS.

Step 3: substitute the vector $\left\{\xi_{1}, \ldots, \xi_{i}, \ldots, \xi_{M}\right\}$ in the $\mathrm{K}-\mathrm{L}$ expansion (Equation 3) to obtain the first realisation of the random field. Then, use the deterministic model to calculate the corresponding response.

Step 4: repeat steps 2 and 3 until obtaining a prescribed number $N_{s}$ of realisations of the random field and their corresponding responses. Then, evaluate the corresponding values of the performance function to obtain the vector $G_{0}=\left\{G_{0}^{1}, \ldots, G_{0}^{k}, \ldots, G_{0}^{N_{s}}\right\}$. Notice that the subscripts ' 0 ' refer to the first level (level 0) of subset simulation. Step 5: prescribe a constant intermediate failure probability $P\left(F_{j}\right)$ for all the failure regions $F_{j}$ and evaluate the first failure threshold $C_{1}$ which corresponds to the failure region $F_{1}$ where $C_{1}$ is equal to the $\left[\left(N_{s} \times P\left(F_{j}\right)\right)+1\right]^{\text {th }}$ value in the increasing list of elements of the vector $G_{0}$. This ensures that the value of $P\left(F_{1}\right)$ will be equal to the prescribed value of $P\left(F_{j}\right)$. Step 6: among the $N_{s}$ realisations, there are $\left[N_{s} \times \mathrm{P}\left(F_{j}\right)\right]$ ones whose values of the performance function are less than $C_{1}$ (i.e. they are located in the failure region $F_{1}$ ). The corresponding vectors $\left\{\xi_{1}, \ldots\right.$, $\left.\xi_{i}, \ldots, \xi_{M}\right\}$ of these realisations are used as "mother vectors' to generate additional $\left[\left(1-\mathrm{P}\left(F_{j}\right)\right) N_{s}\right]$ vectors of $\left\{\xi_{1}, \ldots, \xi_{i}, \ldots, \xi_{M}\right\}$ using Markov chain method based on M-H algorithm. These new vectors are substituted in Equation (3) to obtain the random field realisations of level 1 .

Step 7: the values of the performance function corresponding to the realisations obtained from step 6 are listed in an increasing order and are gathered in the vector of performance functions $G_{1}=\left\{G_{1}^{1}, \ldots, G_{1}^{k}, \ldots, G_{1}^{N_{s}}\right\}$.

Step 8: evaluate the second failure threshold $C_{2}$ as the $\left[\left(N_{s} \times \mathrm{P}\left(F_{j}\right)\right)+1\right]^{\text {th }}$ value in the increasing list of the vector $G_{1}$. 
Step 9: repeat steps 6-8 to evaluate the failure thresholds $C_{3}, C_{4}, \ldots, C_{m}$ corresponding to the failure regions $F_{3}, F_{4}, \ldots, F_{m}$. Notice that contrary to all other thresholds, the last failure threshold $C_{m}$ is negative. Thus, $C_{m}$ is set to zero and the conditional failure probability of the last level $\left[P\left(F_{m} \mid F_{m-1}\right)\right]$ is calculated as:

$$
P\left(F_{m} \mid F_{m-1}\right)=\frac{1}{N_{s}} \sum_{k=1}^{N_{s}} I_{F_{m}}\left(s_{k}\right)
$$

where $I_{F_{m}}=1$ if the performance function $\mathrm{G}\left(s_{k}\right)$ is negative and $I_{F_{m}}=0$ otherwise.

Step 10: Finally, the failure probability $P(F)$ is evaluated according to Equation (6).

It should be mentioned that, in this article, a normal PDF was used as a target probability distribution $\mathrm{P}_{\mathrm{t}}$ (i.e. it was used to generate the $N_{s}$ realisations of the first level of the subset simulation, i.e., level 0). However, a uniform PDF was used as a proposal probability distribution $\mathrm{P}_{\mathrm{p}}$ (i.e. for generating $N_{s}$ realisations for each one of the subsequent levels $1, \ldots, j, \ldots, m)$. The intermediate failure probability $P\left(F_{j}\right)$ of a given level $j(j=0, \ldots, m)$ was chosen equal to 0.1 . Notice that the choice of the value of $P\left(F_{j}\right)$ affects the number of levels $m$ required to reach the limit state surface $G=0$ but its effect on the total number $N_{t}$ of realisations required to calculate the failure probability is very small. If $P\left(F_{j}\right)$ is large, the sequence of failure thresholds $C_{1}$, $\ldots, C_{j}, \ldots C_{m}$ will decrease slowly and a large number of levels will be required to reach the target failure region $F$. In this case, the required number of relisations per level is small. On the contrary, if the $P\left(F_{j}\right)$ value is small, the sequence of failure thresholds $C_{1}, \ldots, C_{j}, \ldots C_{m}$ will reach the target failure region $F$ quickly and a small number of levels will be required. In this case, the required number of realisations per level is large. As a conclusion, an arbitrary value of $P\left(F_{j}\right)$ can be considered for the probabilistic analysis with a small effect on the computational time.

\section{Probabilistic analysis of strip footings}

The probabilistic analysis of shallow foundations resting on a spatially varying soil has been extensively considered in the literature (e.g. Fenton and Griffiths 2003, Popescu et al. 2005, Babu et al. 2006, Griffiths et al. 2006 and Soubra et al. 2008 at ULS and Fenton and Griffiths 2002, 2005 and Fenton et al. 2003 at SLS). These authors have used MCS methodology to perform the probabilistic analysis. In these studies, the mean value and the standard deviation of the system response were extensively investigated. This was not the case for the failure probability because
MCS methodology requires a large number of calls of the deterministic model to accurately calculate a small failure probability.

This article presents a probabilistic analysis at SLS of a strip footing resting on a spatially random soil using subset simulation approach. The objective is the computation of the probability $P_{e}$ of exceeding a tolerable vertical displacement under a prescribed footing load. A footing of breadth $b=2 \mathrm{~m}$ and subjected to a central vertical load $P=1000 \mathrm{kN} / \mathrm{m}$ (i.e. an applied uniform vertical pressure $q_{a}=500 \mathrm{kN} /$ $\mathrm{m}^{2}$ ) was considered in the analysis. The Young's modulus was modelled by a random field and it was assumed to follow a log-normal probability density function. The mean value and the coefficient of variation of the Young's modulus were respectively $\mu_{E}=60 \mathrm{MPa}$ and $\mathrm{COV}_{E}=15 \%$. An exponential covariance function (Equation 4) was used in this article to represent the correlation structure of the random field. The random field was discretised using the K-L expansion. Although an isotropic random field is often assumed in the literature (e.g. Fenton and Griffiths 2002, 2005, Fenton et al. 2003), the vertical autocorrelation length tends to be shorter than the horizontal one due to the geological soil formation process for most natural soil deposits (Cho and Park 2010). A common ratio of about 1-10 for these autocorrelation lengths can be used (Baecher and Christian 2003). Notice, however, that in this article, other values of this ratio were studied and analysed in order to explore some interesting features related to the autocorrelation lengths.

The performance function used to calculate the probability $P_{\mathrm{e}}$ of exceeding a tolerable vertical displacement was defined as follows:

$$
G=v_{\max }-v
$$

where $v_{\max }$ is a prescribed tolerable vertical displacement of the footing centre and $v$ is the vertical displacement of the footing centre due to the applied pressure $q_{a}$. The deterministic model used to calculate the footing vertical displacement $v$ was based on the commercial numerical code FLAC. For this calculation, a footing of width $b$ that rests on a soil domain of width $\mathrm{B}$ and depth $\mathrm{H}$ was considered in the analysis (Figure 2). In contrast to the case of random variables where only one-half of the soil domain (and consequently one-half of the footing) shown in Figure 2 may be considered in the analysis, the entire soil domain shown in Figure 2 was considered in this article. This is because the random field creates non-symmetrical soil movements. An optimal non-uniform but symmetrical mesh composed of 750 zones was employed. In order to accurately calculate the footing displacement, the 


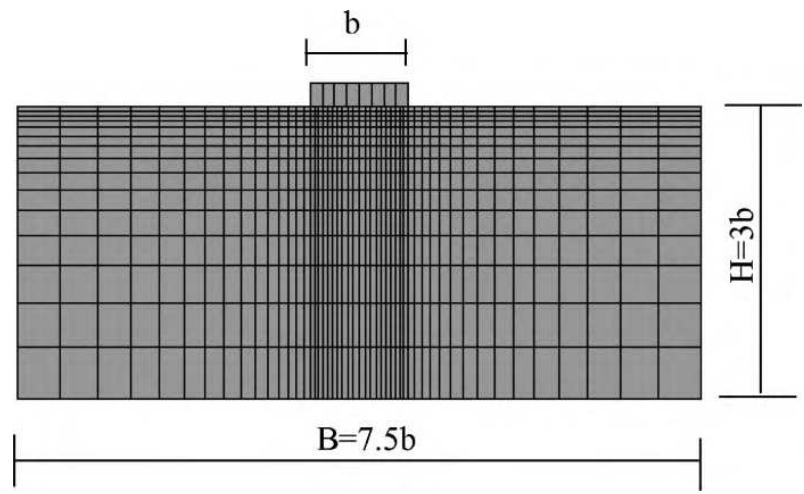

Figure 2. Soil domain and mesh used in the numerical simulation.

mesh was refined near the footing edges where high stress gradient may occur. For the displacement boundary conditions, the bottom boundary was assumed to be fixed and the vertical boundaries were constrained in motion in the horizontal directions. Although this article presents an SLS analysis, the soil behaviour was modelled by a conventional elasticperfectly plastic model based on Mohr-Coulomb failure criterion in order to take into account the possible plastification that may occur near the edges of the foundation even under the service loads. The strip footing was modelled by a linear elastic model. It is connected to the soil via interface elements. The values of the different parameters of the soil, footing and interface are given in Table 1.

In order to calculate the footing vertical displacement for a given realisation, (1) the vertical and horizontal coordinates of the centre of each element of the mesh were calculated; then, Equation (3) was used to calculate the value of the Young's modulus at the centre of each element, (2) geostatic stresses were applied to the soil, (3) the obtained displacements were set to zero in order to obtain the footing displacement due to only the footing applied pressure and (4) the uniform vertical pressure was applied to the footing and the vertical displacement at the footing centre due to this pressure was calculated.

Table 1. Shear strength and elastic properties of soil, footing, and interface.

\begin{tabular}{lccc}
\hline Variable & Soil & Footing & Interface \\
\hline$C$ & $20 \mathrm{kPa}$ & N/A & $20 \mathrm{kPa}$ \\
$\varphi$ & $30^{\circ}$ & N/A & $30^{\circ}$ \\
$\psi_{\mathrm{s}}=2 / 3 \varphi$ & $20^{\circ}$ & N/A & $20^{\circ}$ \\
$E$ & $60 \mathrm{MPa}$ & $25 \mathrm{GPa}$ & $\mathrm{N} / \mathrm{A}$ \\
$v$ & 0.3 & 0.4 & $\mathrm{~N} / \mathrm{A}$ \\
$K_{\mathrm{n}}$ & $\mathrm{N} / \mathrm{A}$ & $\mathrm{N} / \mathrm{A}$ & $1 \mathrm{GPa}$ \\
$K_{\mathrm{s}}$ & $\mathrm{N} / \mathrm{A}$ & $\mathrm{N} / \mathrm{A}$ & $1 \mathrm{GPa}$ \\
\hline
\end{tabular}

\section{Numerical results}

This section aims at presenting the probabilistic numerical results. It is organised as follows: (1) the minimal number $M$ of terms of the K-L expansion corresponding to a prescribed accuracy was determined, (2) the optimal number of realisations $N_{s}$ per level of subset simulation was chosen, (3) a parametric study to investigate the effect of the horizontal and vertical autocorrelation lengths of the random field and the coefficient of variation of this random field on $P_{\mathrm{e}}$ was presented and discussed and (4) a reliabilitybased design and analysis of strip footings based on some fragility curves was presented and discussed.

It should be mentioned that all subsequent probabilistic results are presented based on nondimensional autocorrelation lengths $L_{\ln x}$ and $L_{\ln y}$ where $\mathrm{L}_{\ln x}=l_{\ln x} / \mathrm{b}$ and $L_{\ln y}=l_{\ln y} / \mathrm{b}$. The nondimensionality of $L_{\ln x}$ and $L_{\ln y}$ was verified numerically for different cases and was found to be valid when the ratio between the footing width and the depth of the soil domain $\mathrm{b} / \mathrm{H}$ is constant.

\section{Optimal size of the $K-L$ expansion}

As mentioned earlier, the accuracy of the approximated random field depends on the size of the K-L expansion (i.e. the number of terms $M$ ). Figure 3 presents the error estimate of the approximated random field for the most critical configurations of $L_{\ln x}$ and $L_{\ln y}$ used in this article, that is, for those requiring greater number of terms in the K-L expansion. These configurations correspond (as will be shown later) to $\left[\left(L_{\ln x}=5\right.\right.$ and $\left.L_{\ln y}=0.25\right)$ and $\left(L_{\ln x}=2.5\right.$ and $\left.\left.L_{\ln y}=0.5\right)\right]$ in case of anisotropic random field and $\left[\left(L_{\ln x}=L_{\ln y}=0.5\right)\right.$ and $\left(L_{\ln x}\right.$ $\left.\left.=L_{\ln y}=1.5\right)\right]$ in case of isotropic random field. Figure 3 indicates that the error estimate decreases with the increase in the number of terms of the K-L expansion. From this figure, for $M=100$ terms, the error estimate is less than $13 \%$ for the previously mentioned cases except for the case of $L_{\ln x}=L_{\ln y}=0.5$ where $M \approx 500$ terms are required to obtain such a small error. Notice that the configurations used in this article correspond to $L_{\ln x}$ and $L_{\ln y}$ values equal to or greater than the aforementioned configurations. As a conclusion, the number of terms of the K-L expansion will be set to $M=100$ terms for all the probabilistic calculations presented in this article except for the case of the isotropic random field when $L_{\ln x}$ and $L_{\ln y}$ are less than 1.5 where $M$ will be set equal to 500 terms. This ensures that for all the configurations considered in this article, the error will be less than $13 \%$. 


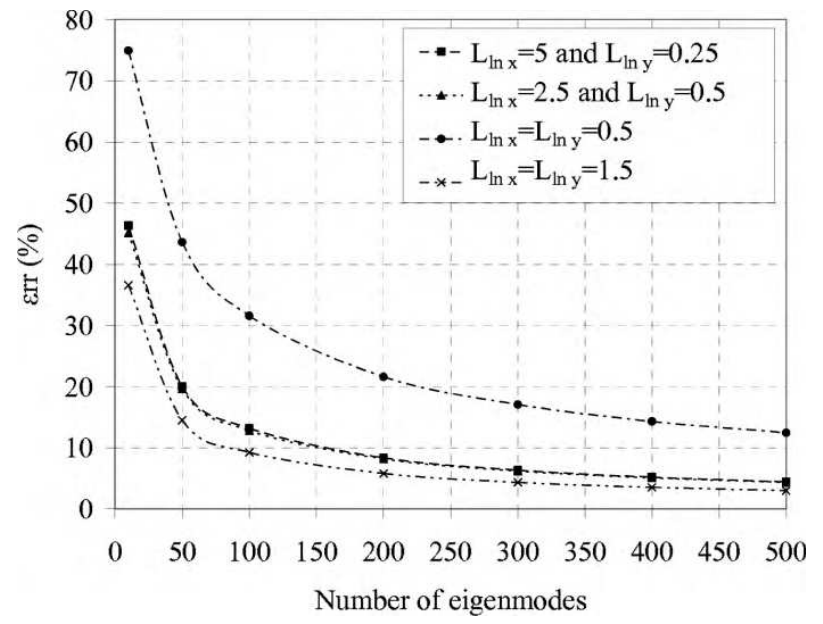

Figure 3. Error estimate versus the number of eigenmodes for different values of $L_{\ln x}$ and $L_{\ln y}$ when $\mathrm{H} / \mathrm{b}=3$.

\section{Selection of the optimal number of realisations $\mathrm{N}_{\mathrm{s}}$ per level of subset simulation}

The number of realisations $N_{s}$ to be used per level of subset simulation should be sufficient to accurately calculate the $P_{\mathrm{e}}$ value. This number should be greater than 100 to provide a small bias in the calculated $P_{\mathrm{e}}$ value (Honjo 2008). In order to determine the optimal number of realisations $N_{s}$ to be used per level, different values of $N_{s}(50,100,150,200$ and 250 realisations) were considered to calculate $P_{\mathrm{e}}$. For each $N_{s}$ value, $P_{\mathrm{e}}$ computed by subset simulation was compared to that obtained by MCS methodology using $N=20,000$ realisations. The comparison was carried out in Figure 4 at the different levels, that is, at the different failure threshold $C_{j}$ of the subset simulation approach). A random field with $L_{\ln x}=5$ and $L_{\ln y}=0.5$ (called hereafter the reference case) was considered herein. Notice that the failure thresholds $C_{j}$ of the different levels of the subset simulation were calculated and presented in Table 2 for the aforementioned values of $N_{s}$. This table indicates that the failure threshold decreases with the successive levels until reaching a negative value at the last level which means that the realisations generated by the subset simulation successfully progress towards the limit state surface $G=0$. Notice that for a given $N_{s}$ value, the computation of $P_{\mathrm{e}}$ at a given level $j$ of the subset simulation is performed by using Equation (6). However, in order to calculate $P_{\mathrm{e}}$ at this level by MCS methodology, the performance function is set equal to the corresponding failure threshold $C_{j}$. In this case, the failure region is defined as $G \leq C_{j}$ and the safety region is defined as $G>C_{j}$. Thus, the value of $P_{\mathrm{e}}$ at a given level $j$ can be calculated as:

$$
P\left(F_{j}\right)=\frac{1}{N} \sum_{k=1}^{N} I_{F_{j}}\left(G_{k}\right)
$$

where $G_{k}$ is the value of the performance function corresponding to the $k$ th realisation of MCS, $I_{F}=1$ if $G_{k}<C_{j}$ and $I_{F}=0$ otherwise.

Figure $4 \mathrm{a}$ shows that for the case where $N_{s}=50$ realisations, $P_{\mathrm{e}}$ calculated by subset simulation is different from that computed by MCS for the different levels of subset simulation. This observation is in conformity with the recommendation by Honjo (2008) who suggested that $N_{s}$ value should be at least equal to 100 . The difference between the $P_{\mathrm{e}}$ values calculated by subset simulation and those computed by MCS becomes smaller for larger $N_{s}$ values (Figure $4 \mathrm{~b}-\mathrm{e})$. For the cases where $N_{s} \geq 200$ realisations (Figure $4 \mathrm{~d}, \mathrm{e}$ ), the failure probabilities calculated by subset simulation were found to be very close to those computed by MCS methodology for the different levels of subset simulation. Consequently, $N_{s}=200$ realisations will be considered in the subsequent probabilistic calculations. In this case, the final $P_{\mathrm{e}}$ value [i.e. $P\left(F_{m}\right)$ which corresponds to $C=0$ was equal to $\left.3.78 \times 10^{-4}\right]$. This value is to be compared to the value of $3.8 \times 10^{-4}$ given by MCS. It should be mentioned here that, since $\mathrm{P}\left(F_{j}\right)$ was chosen to be equal to 0.1 , four levels of subset simulation were found necessary to arrive at the limit state surface $G=0$ as may be seen from Table 2. Therefore, when $N_{s}=200$ realisations, a total number of realisations $N_{t}=200 \times 4=800$ realisations were required to calculate the final $P_{\mathrm{e}}$ value. In this case, the coefficient of variation of $P_{\mathrm{e}}$ computed by subset simulation was determined using the following equation:

$$
\operatorname{COV}_{P_{e}}=\sum_{j=1}^{m}\left[\operatorname{COV}_{P\left(F_{j}\right)}\right]^{2}
$$

where $\operatorname{COV}_{P\left(F_{j}\right)}$ is the coefficient of variation at an intermediate level $j$. Details on the computation of $\operatorname{COV}_{\mathrm{P}\left(F_{j}\right)}$ may be found in Au and Beck $(2001,2003)$. When using 800 realisations, the COV of $P_{\mathrm{e}}$ computed by subset simulation is equal to 0.51 . However, if one uses MCS with the same number of realisations (i.e. 800 realisations), the value of $\mathrm{COV}$ of $P_{\mathrm{e}}$ is equal to 1.81 . This means that for the same computational effort, the subset simulation approach provides a smaller value of $C O V_{P_{e}}$ than MCS.

Although the computation time of the 20,000 realisations by MCS is significant (about 70 days), 


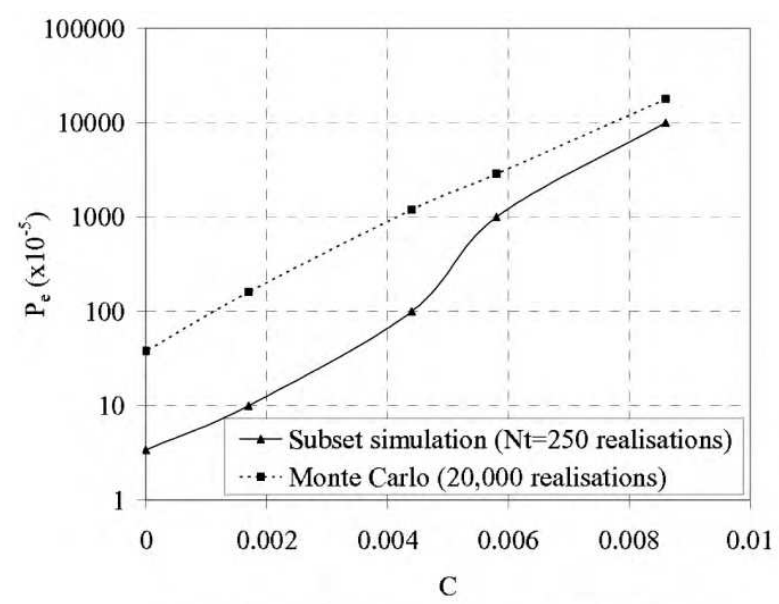

a. $\mathrm{N}_{\mathrm{s}}=50$ realisations per level

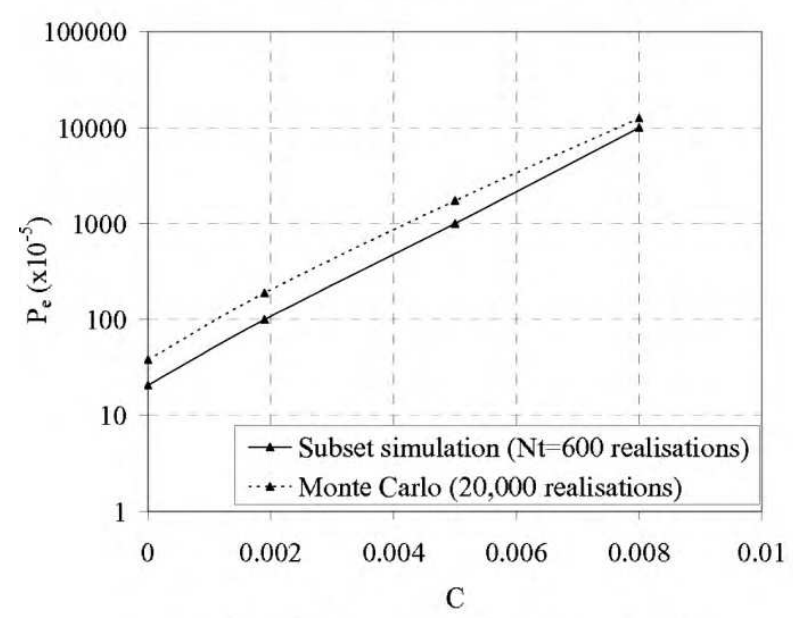

c. $\mathrm{N}_{\mathrm{s}}=150$ realisations per level

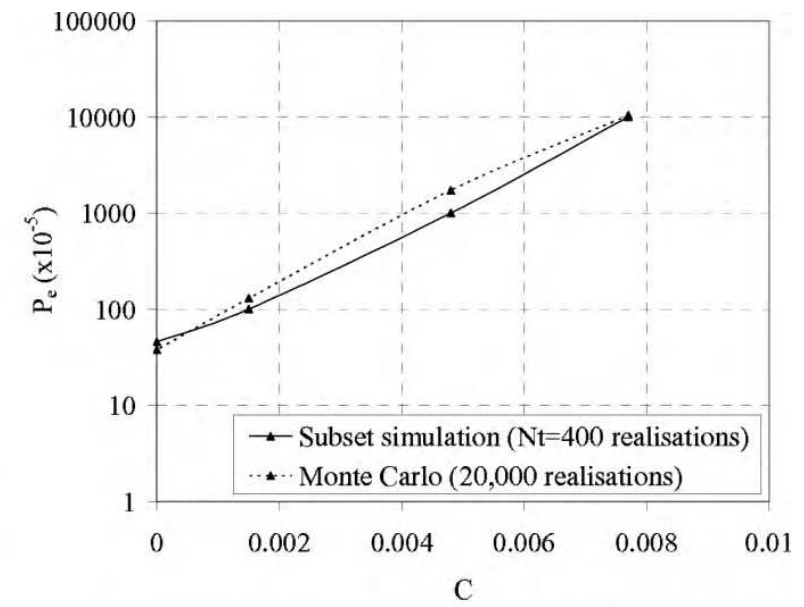

b. $\mathrm{N}_{\mathrm{s}}=100$ realisations per level

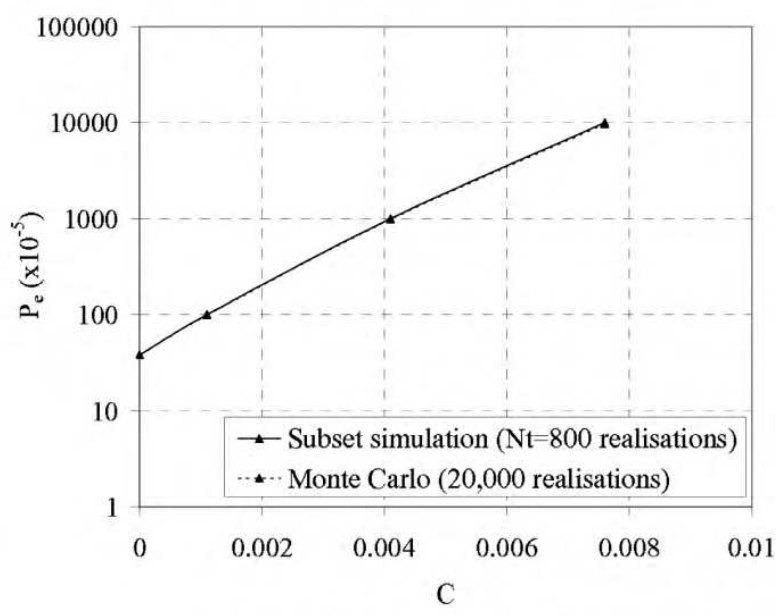

d. $\mathrm{N}_{\mathrm{s}}=200$ realisations per level

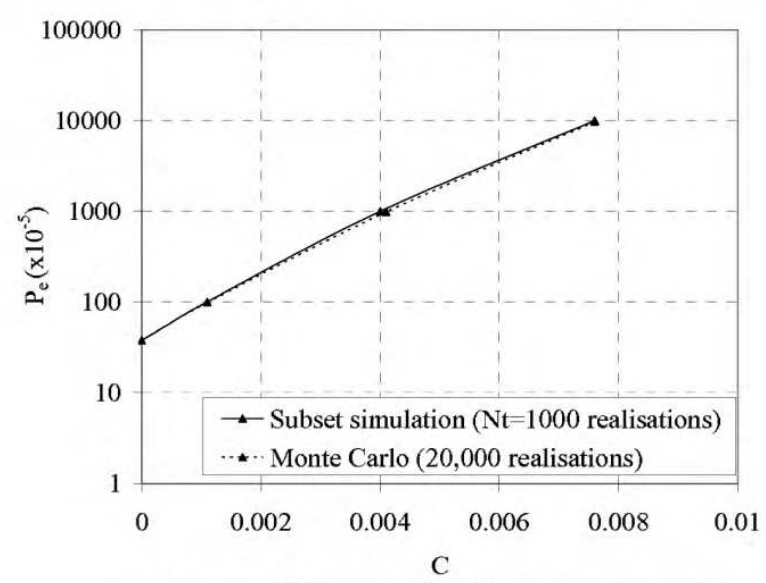

e. $\mathrm{N}_{\mathrm{s}}=250$ realisations per level

Figure 4. Comparison between the $P_{\mathrm{e}}$ values obtained by subset simulation and those obtain by MCS for five values of $N_{s}\left(v_{\max } / \mathrm{b}=2 \times 10^{-2}\right.$ and $\left.q_{a}=500 \mathrm{kN} / \mathrm{m}^{2}\right)$.

this number of realisations remains insufficient to assure an accurate $P_{\mathrm{e}}$ value with a small value of $\mathrm{COV}_{P_{e}}$. In this article, the $\operatorname{COV}_{P_{e}}$ for 20,000 realisations by MCS was found approximately 0.4 . As an alternative approach, one may determine the optimal $N_{S}$ value by comparing the $P_{\mathrm{e}}$ values given by 
Table 2. Evolution of the failure threshold $C_{j}$ with the different levels $\mathrm{j}$ of the subset simulation and with the number of realisations $\left(N_{s}\right)$ per level

\begin{tabular}{lcccrr}
\hline & \multicolumn{4}{c}{ Number of realisations per level $\left(N_{s}\right)$} \\
\cline { 2 - 5 } Failure threshold $C_{j}$ for each level $j$ & 50 & 100 & 150 & 200 & 250 \\
\hline $\mathrm{C}_{1}$ & 0.0086 & 0.0077 & 0.0080 & 0.0076 & 0.0076 \\
$\mathrm{C}_{2}$ & 0.0058 & 0.0048 & 0.0050 & 0.0041 & 0.0040 \\
$\mathrm{C}_{3}$ & 0.0044 & 0.0015 & 0.0019 & 0.0011 & -0.0011 \\
$\mathrm{C}_{4}$ & 0.0017 & -0.0019 & -0.0007 & -0.0020 & -0.0018 \\
$\mathrm{C}_{5}$ & -0.0015 & - & - & - \\
\hline
\end{tabular}

subset simulation for increasing $N_{s}$ values. The $N_{s}$ value beyond which $P_{\mathrm{e}}$ converges (i.e. slightly varies with the increase of $N_{s}$ ) is the optimal $N_{s}$ value. In the present analysis, it was found that $P_{\mathrm{e}}$ converges when $N_{s}=200$ realisations. This is because the final $P_{\mathrm{e}}$ values (corresponding to $C=0$ ) are respectively equal to $3.78 \times 10^{-4}$ and $3.81 \times 10^{-4}$ for $N_{s}=200$ and 250 realisations. The corresponding values of $\mathrm{COV}_{P_{e}}$ are equal to 0.51 and 0.43 which indicates (as expected) that the $\operatorname{COV}_{P_{e}}$ decreases with the increase in the number of realisations. As a conclusion, this alternative procedure is recommended to determine the optimal $N_{s}$ value for the probabilistic analysis based on subset simulation approach.

\section{Parametric study}

In this section, a parametric study is performed to investigate the effect of the soil variability (coefficient of variation and autocorrelation lengths of the Young's modulus) on $P_{\mathrm{e}}$.

Figure 5 shows the effect of the autocorrelation length on $P_{\mathrm{e}}$ in the case of an isotropic random field. This figure also shows (for the same value of the coefficient of variation) the value of $P_{\mathrm{e}}$ corresponding to the case of a homogeneous soil. In this case, $P_{\mathrm{e}}$ was calculated based on the assumption that, for a given realisation, each element of the deterministic grid was affected the same random value of the Young's modulus (i.e. the Young's modulus was modelled as a random variable and not as a random field). Figure 5 indicates that the increase in the autocorrelation length $\left(L_{\ln x}=L_{\ln y}\right)$ increases the $P_{\mathrm{e}}$ value. However, the rate of increase decreases for the large autocorrelation lengths. This is because the random field tends to the case of a homogeneous soil for great values of the autocorrelation lengths $\left(L_{\ln x}=L_{\ln y}>50\right)$ as shown in Figure 6a. For smaller values of the autocorrelation length, one obtains a soil heterogeneity which results in a variety of values of the Young's modulus beneath the footing (Figure 6b). In this case, the soil under the footing contains some zones with high values of the Young's modulus and other zones with small values of the Young's modulus (i.e. a mixture of stiff zones and soft zones). Due to the high footing rigidity, the footing displacement is resisted by the stiff soil zones under it; the soft soil zones under the footing being of little effect in this case. This leads to a small value of the footing vertical displacement and consequently to a small value of $P_{\mathrm{e}}$. As a conclusion, for a given value of the coefficient of variation, modelling the Young's modulus as a random variable rather than a random field is conservative (Fenton and Griffiths 2002, 2005). This is because the settlement predicted when assuming a homogeneous soil is larger than that of a real soil for which the parameters vary spatially.

In order to investigate the effect of the anisotropy of the random field, $P_{\mathrm{e}}$ was computed and plotted versus the horizontal and the vertical autocorrelation lengths $\left(L_{\ln x}\right.$ and $\left.L_{\ln y}\right)$ in Figure 7 and 8, respectively. Both figures show that $P_{\mathrm{e}}$ presents a maximum value at a certain ratio of $L_{\ln x}$ to $L_{\ln y}$. This observation can be explained as follows:

Referring to Figure 7, the very small value of $L_{\ln x}$ creates a vertical multilayer composed of thin

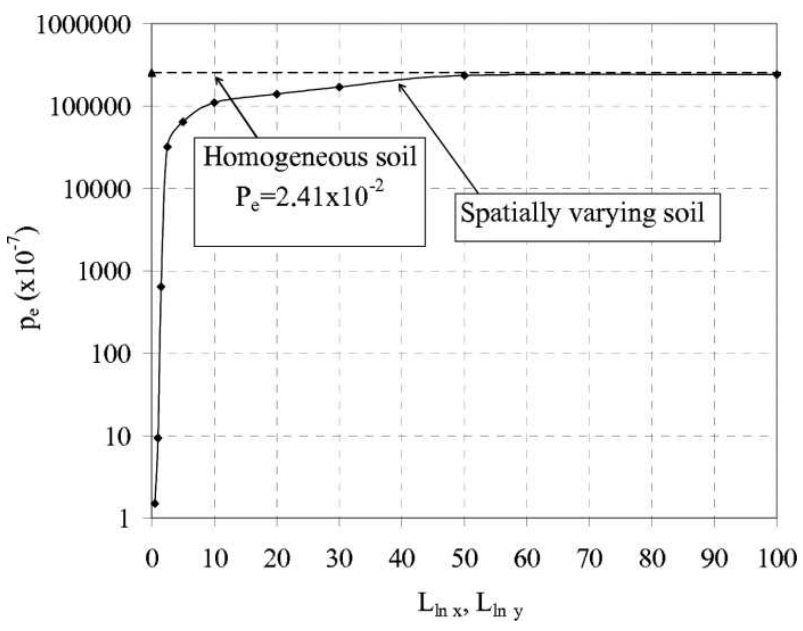

Figure 5. Effect of the autocorrelation length on $P_{\mathrm{e}}$ in case of an isotropic random field $\left(v_{\max } / \mathrm{b}=2 \times 10^{-2}\right.$ and $q_{a}=500$ $\mathrm{kN} / \mathrm{m}^{2}$ ). 


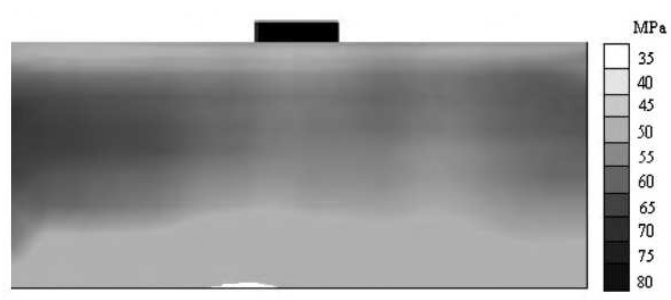

a. $\mathrm{L}_{\ln \mathrm{x}}=\mathrm{L}_{\ln \mathrm{y}}=100$

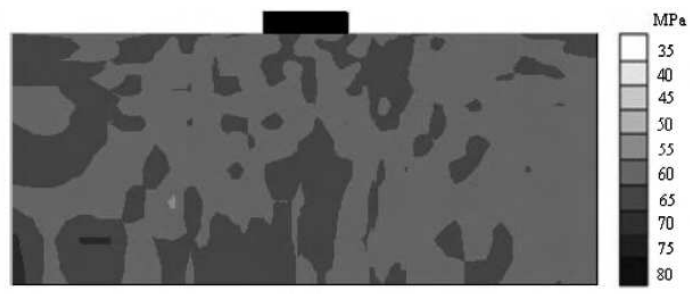

b. $\mathrm{L}_{\ln x}=\mathrm{L}_{\ln \mathrm{y}}=0.1$

Figure 6. Gray-scale representation of the random field for two values of the autocorrelation length in case of an isotropic random field.

sub-layers each of which may have either a high or a small value of the Young's modulus (Figure 9a). This variety of sub-layers leads to a small footing displacement and consequently to a small value of $P_{\mathrm{e}}$. The small footing displacement occurs because the rigid footing is resisted by the sub-layers having high values of Young's modulus beneath it; the sub-layers having small values of Young's modulus being of little effect in this case. However, when $L_{\ln x}$ is very large, the problem becomes similar to that of the 1D vertical random field. In this case, the $P_{\mathrm{e}}$ value is smaller than that of the $2 \mathrm{D}$ case. This is because, for large values of $L_{\ln x}$, one obtains a horizontal multilayer for which each sub-layer may have either a high or a small value of the Young's modulus (Figure 9b). The sub-layers having high values of Young's modulus reduce the footing displacement and thus lead to smaller values of $P_{\mathrm{e}}$. To check the fact that $P_{\mathrm{e}}$ tends to that corresponding to a $1 \mathrm{D}$ random field as $L_{\ln x}$ gets larger and approaches infinity, the $P_{\mathrm{e}}$ corresponding to the $1 \mathrm{D}$ vertical random field and that corresponding to $L_{\ln x}=5000$ were calculated for the three cases considered in Figure 7 (i.e. for $L_{\ln y}=0.5$, 1 and 1.5). These $P_{\mathrm{e}}$ values were compared to those

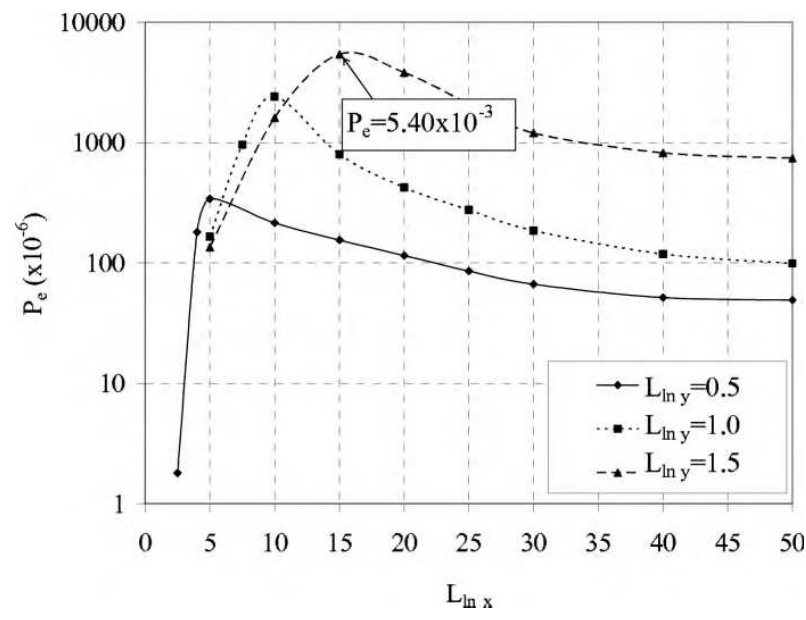

Figure 7. Effect of the horizontal autocorrelation length on $P_{\mathrm{e}}$ for different values of $L_{\ln y}\left(v_{\max } / \mathrm{b}=2 \times 10^{-2}\right.$ and $q_{\mathrm{a}}=500 \mathrm{kN} / \mathrm{m}^{2}$ ). corresponding to the 2D random field in Table 3 . These results confirm that $P_{\mathrm{e}}$ tends to the value corresponding to the $1 \mathrm{D}$ case as $L_{\ln x}$ gets larger and approaches infinity. Finally, for medium values of $L_{\ln x}$, the soil contains a number of stiff zones adjacent to a number of soft zones whose areas are less extended than those corresponding to the case of small and high values of $L_{\ln x}$ (Figure 9e). This leads to a larger footing displacement. As a result, $P_{\mathrm{e}}$ reaches its maximum value for these intermediate values of $L_{\ln x}$.

Referring to Figure 8, when $L_{\ln y}$ is very small, one obtains a horizontal multilayer composed of thin sublayers (Figure 9c). However, the large value of $L_{\ln y}$ creates a vertical multilayer and makes the random field tend to the case of 1D horizontal random field (Figure 9d). For medium values of $L_{\ln y}$, the soil is composed of some zones with high values of Young's modulus and other zones with small values of Young's modulus (Figure 9e). For the three cases of small, intermediate or high values of $L_{\ln y}$, the same explanation given before for Figure 7 remains valid herein.

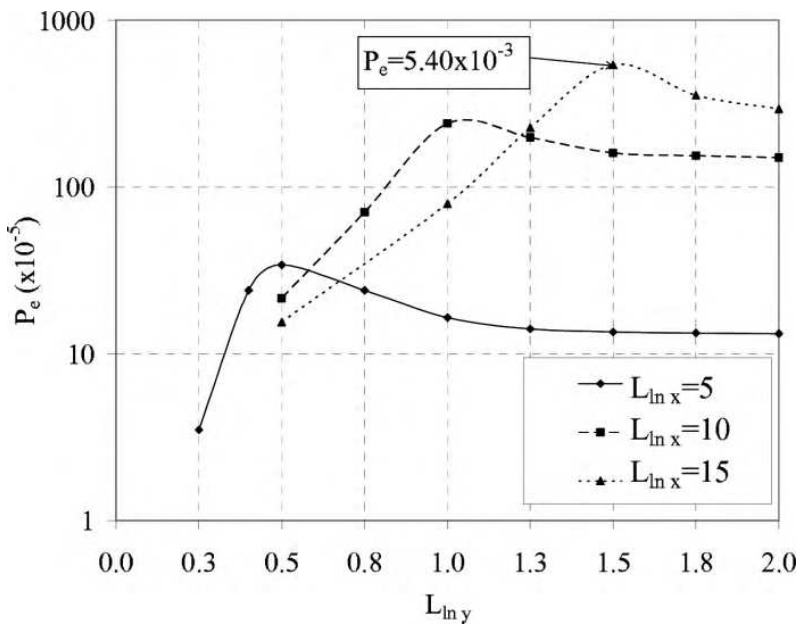

Figure 8. Effect of the vertical autocorrelation length on $P_{\mathrm{e}}$ for different values of $L_{\ln x}\left(v_{\max } / \mathrm{b}=2 \times 10^{-2}\right.$ and $q_{a}=500$ $\mathrm{kN} / \mathrm{m}^{2}$ ). 


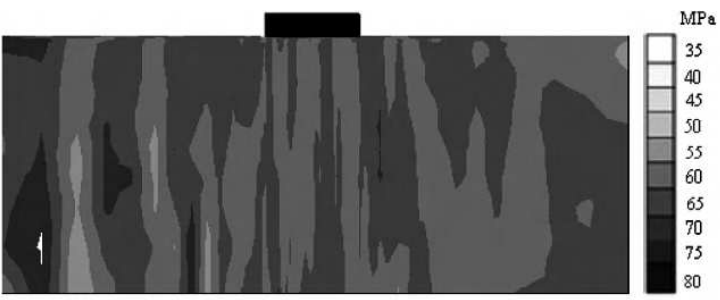

a. $\mathrm{L}_{\ln \mathrm{x}}=0.1$ and $\mathrm{L}_{\ln \mathrm{y}}=1.5$

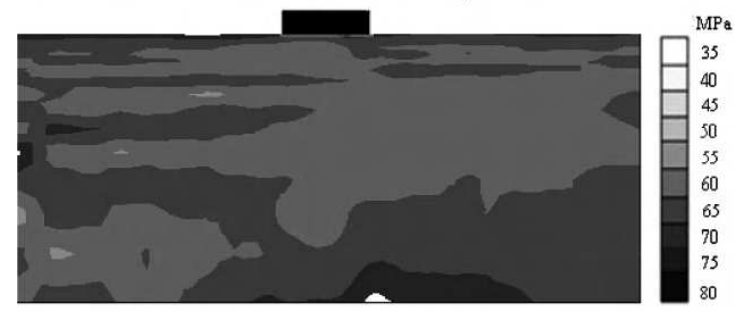

c. $\mathrm{L}_{\ln x}=1.5$ and $\mathrm{L}_{\ln \mathrm{y}}=0.1$

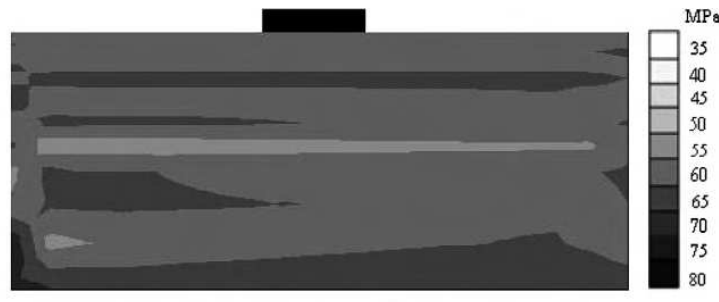

b. $\mathrm{L}_{\ln \mathrm{x}}=5000$ and $\mathrm{L}_{\ln \mathrm{y}}=0.1$

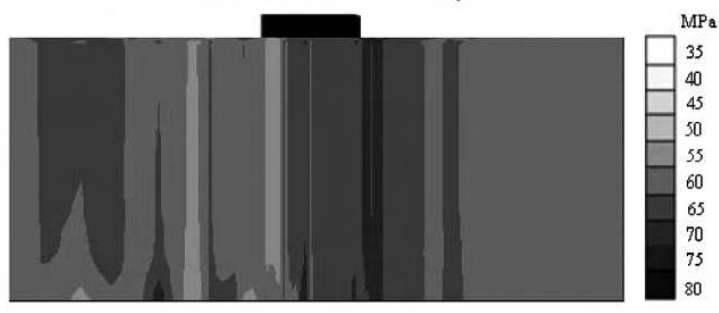

d. $L_{\ln x}=0.1$ and $L_{\ln y}=5000$

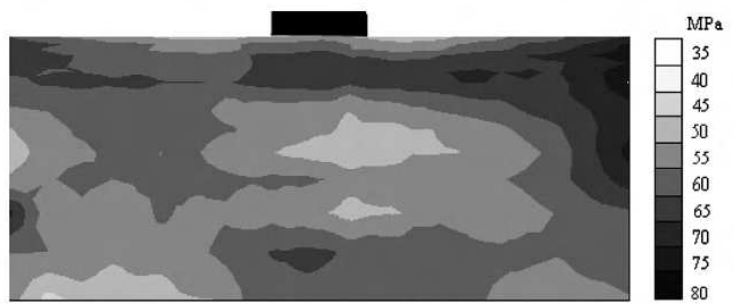

e. $\mathrm{L}_{\ln \mathrm{x}}=5$ and $\mathrm{L}_{\ln \mathrm{y}}=0.5$

Figure 9. Gray-scale representation of the random field for different values of the autocorrelation lengths in case of an anisotropic random field.

As a conclusion, the soil configuration gradually changes from a vertical to a horizontal multilayer as $L_{\ln x}$ increases. Similarly, the soil configuration gradually changes from a horizontal to a vertical multilayer as $L_{\ln y}$ increases. The footing vertical displacement is the largest for medium values of $L_{\ln x}$ or $L_{\ln y}$ where the soil movements may occur more easily. Consequently, the $P_{\mathrm{e}}$ presents a maximum value in this case.

Notice that the ratio of $L_{\ln x} / L_{\ln y}$ for which $P_{\mathrm{e}}$ is maximum depends on the values of the soil and footing parameters (i.e. $\mu_{E}, v, c, \varphi, b$ ). For the case studied herein, this ratio is equal to 10 . Notice finally that for the same ratio of $L_{\ln x} / L_{\ln y}$ but greater values

Table 3. Effect of $L_{\ln x}$ on $P_{\mathrm{e}}$ for different values of $L_{\ln y}$.

\begin{tabular}{lccc}
\hline & \multicolumn{1}{c}{$P_{\mathrm{e}}$} \\
\cline { 2 - 4 }$L_{\ln x}$ & $L_{\ln y}=0.5$ & $L_{\ln y}=1.0$ & $L_{\ln y}=1.5$ \\
\hline 2.50 & $1.80 \times 10^{-6}$ & - & - \\
4.00 & $1.79 \times 10^{-4}$ & - & - \\
5.00 & $3.41 \times 10^{-4}$ & $1.65 \times 10^{-4}$ & $1.35 \times 10^{-4}$ \\
10.00 & $2.15 \times 10^{-4}$ & $24.00 \times 10^{-4}$ & $1.60 \times 10^{-3}$ \\
15.00 & $1.55 \times 10^{-4}$ & $7.95 \times 10^{-4}$ & $5.40 \times 10^{-3}$ \\
20.00 & $1.15 \times 10^{-4}$ & $4.23 \times 10^{-4}$ & $3.80 \times 10^{-3}$ \\
25.00 & $8.55 \times 10^{-5}$ & $2.75 \times 10^{-4}$ & $2.10 \times 10^{-3}$ \\
30.00 & $6.65 \times 10^{-5}$ & $1.85 \times 10^{-4}$ & $1.20 \times 10^{-3}$ \\
40.00 & $5.15 \times 10^{-5}$ & $1.19 \times 10^{-4}$ & $8.20 \times 10^{-4}$ \\
50.00 & $4.90 \times 10^{-5}$ & $9.88 \times 10^{-5}$ & $7.40 \times 10^{-4}$ \\
5000.00 & $3.20 \times 10^{-5}$ & $5.85 \times 10^{-5}$ & $3.65 \times 10^{-4}$ \\
One-dimensional & $2.45 \times 10^{-5}$ & $4.75 \times 10^{-5}$ & $2.45 \times 10^{-4}$ \\
\hline
\end{tabular}


of $L_{\ln x}$ and $L_{\ln y}$, the maximum value of $P_{\mathrm{e}}$ was found to be higher (Figure 7 and 8 ). This is due to the simultaneous increase of the autocorrelation lengths in both the vertical and the horizontal directions which makes the $P_{\mathrm{e}}$ tend to that corresponding to the case of the random homogeneous soil that does not exhibit spatial variability. In this case, the $P_{\mathrm{e}}$ value is equal to $2.41 \times 10^{-2}$ (Figure 5). This value is greater by more than one order of magnitude with respect to the maximum value of $P_{\mathrm{e}}$ given in Figures 7 and 8 which is equal to $5.4 \times 10^{-3}$. This clearly illustrates the benefit of considering the soil spatial variability in the analysis. The numerical results of Figures 7 and 8 also indicate that $P_{\mathrm{e}}$ is more sensitive to the vertical autocorrelation length. This is because the rate of change in $P_{\mathrm{e}}$ (i.e. rate of increase or decrease) when increasing the vertical autocorrelation length by a certain percentage is larger than that when increasing the horizontal autocorrelation length by the same percentage. For example, the increase of the vertical autocorrelation length by $100 \%$ with respect to the reference case (i.e. $L_{\ln x}=5$ and $L_{\ln \mathrm{y}}=0.5$ ) decreases the value of $P_{\mathrm{e}}$ by $51.6 \%$. However, the increase of the horizontal autocorrelation length by $100 \%$ with respect to the reference case decreases the value of $P_{\mathrm{e}}$ by only $36.9 \%$.

The effect of the coefficient of variation of the Young's modulus on $P_{\mathrm{e}}$ was presented in Figure 10. This figure indicates that, for both cases of isotropic and anisotropic random fields, the increase in the coefficient of variation of the Young's modulus from $10 \%$ to $15 \%$ significantly increases the value of $P_{\mathrm{e}}$. The increase is greater than one order of magnitude for both cases of isotropic and anisotropic autocorrelation lengths. This means that careful experimental

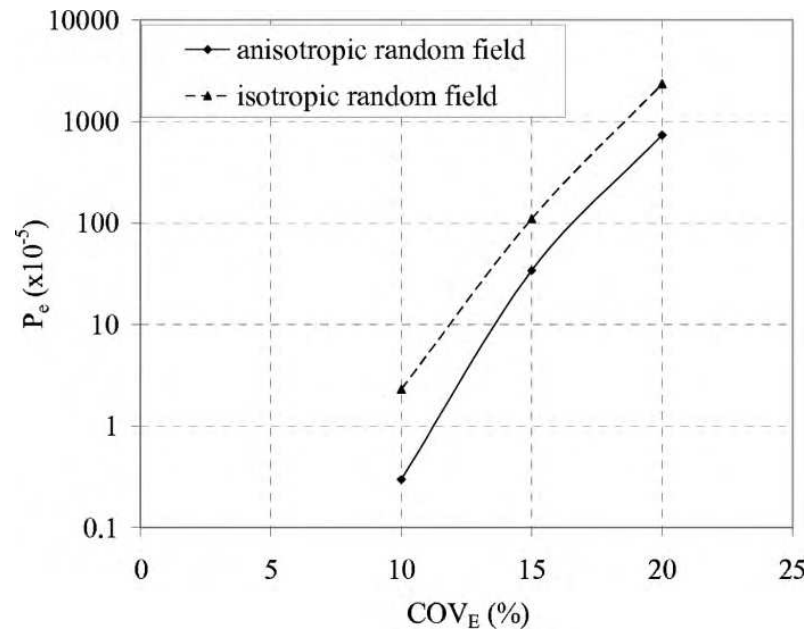

Figure 10. Effect of $\mathrm{COV}_{E}$ on the $P_{\mathrm{e}}$ value in case of (1) anisotropic random field with $L_{\ln x}=5$ and $L_{\ln y}=0.5$ and (2) isotropic random field with $L_{\ln x}=L_{\ln y}=5$. investigations concerning the variability of this parameter are necessary to lead to reliable results.

\section{Reliability-based design and analysis of strip footings}

The probability that a certain level of damage (tolerable vertical displacement) will be exceeded under a given applied footing pressure can be expressed in the form of fragility curves (e.g. Popescu et al. 2005). Figure 11a presents several fragility curves corresponding to three values of Poisson's ratio $(0.25,0.3$ and 0.35 ) and to three levels of damage [(1) minor damage for which $v_{\max } / \mathrm{b}=1.5 \times 10^{-2}$, (2) medium damage for which $v_{\max } / \mathrm{b}=2.0 \times 10^{-2}$ and (3) major damage for which $\left.v_{\max } / \mathrm{b}=2.5 \times 10^{-2}\right]$ for the reference case (i.e. $L_{\ln x}=5$ and $L_{\ln y}=0.5$ ). In this figure, the footing pressure was normalised with respect to the mean value of the Young's modulus

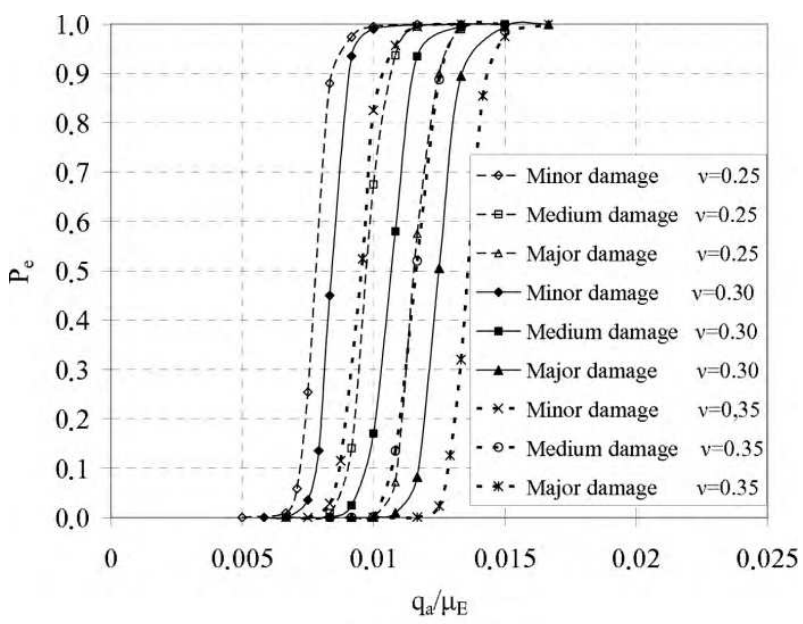

a. Normal scale

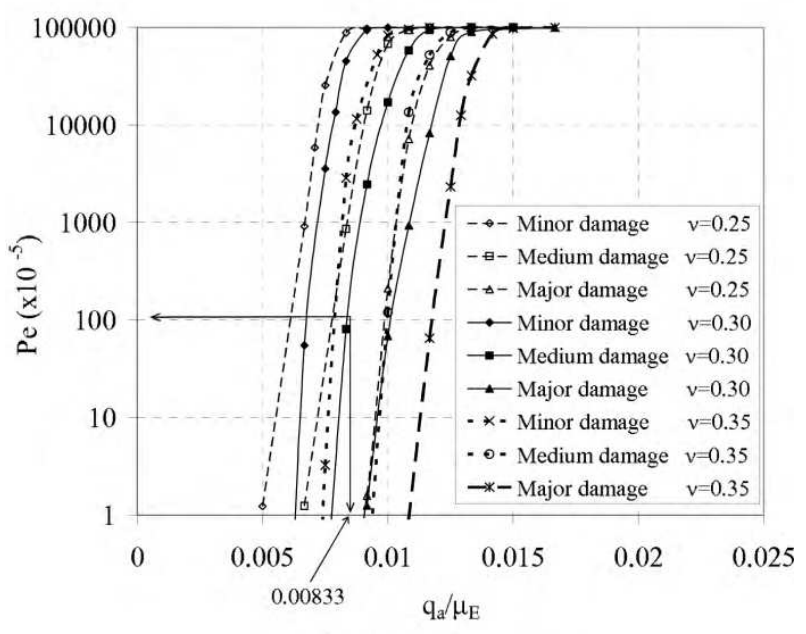

b. Semi-log scale

Figure 11. Fragility curves for different values of Poisson's ratio and different values of the damage level. 
and the three damage levels were normalised with respect to the footing breadth. The curves of Figure 11a can be employed to perform either an SLS probabilistic analysis or an SLS probabilistic design of strip footings. For the probabilistic analysis, this figure allows one to determine the probability of exceeding a tolerable vertical displacement corresponding to a given value of the applied footing pressure, to a given value of $\mu_{E}$, to a given value of Poisson's ratio and to a given value of the prescribed damage level. Concerning the footing design, Figure 11a can be employed to determine the footing pressure (and consequently the footing breadth $b$ ) for a given load, for a given $\mu_{E}$ value, for a given value of Poisson's ratio, for a prescribed damage level and for a target probability of exceeding this damage level. Figure 11a was plotted in a semi-log scale in Figure $11 \mathrm{~b}$ to clearly identify the small $P_{\mathrm{e}}$ values at the distribution tail. As an application example of these curves, if $\mu_{E}=60 \mathrm{MPa}, v=0.3$ and a medium damage with a target $P_{\mathrm{e}}$ value of $10^{-3}$ is allowed, $q_{a}$ $\mu_{E}=0.00833$. Consequently, the footing pressure is $q_{\mathrm{a}}=0.00833 \times 60 \times 10^{3}=500 \mathrm{kN} / \mathrm{m}^{2}$. Hence the probabilistic footing breadth required to support a given footing service load $P_{s}$ can be calculated as $\mathrm{b}=P_{s} / q_{a}$.

\section{Conclusion}

This article aims at presenting a probabilistic analysis at SLS of a strip footing resting on a soil with spatially varying Young's modulus using subset simulation approach. The footing is subjected to a central vertical load. Compared to MCS methodology, the use of subset simulation approach has significantly reduced the number of calls of the deterministic model. The main findings of this article can be summarised as follows:

(1) - In case of isotropic random fields:

- The probability $P_{\mathrm{e}}$ of exceeding a tolerable vertical displacement increases with the increase of the autocorrelation length. For large autocorrelation lengths, $P_{\mathrm{e}}$ was found too close to that of a homogeneous random soil (i.e. that corresponding to the case of a random variable).

(2) - In case of anisotropic random fields:

- $P_{e}$ presents a maximum value for a given ratio of $L_{\ln x} / L_{\ln y}$. For greater values of $L_{\ln x}$ and $L_{\ln y}$, this maximum value of $P_{\mathrm{e}}$ was found to be higher. At the limit, when both $L_{\ln x}$ and $L_{\ln y}$ tend to infinity, one obtains the $P_{\mathrm{e}}$ value corresponding to the case of a random homogeneous soil.
- $P_{\mathrm{e}}$ is more sensitive to the vertical autocorrelation length than the horizontal one.

(3) The increase of the coefficient of variation of the Young's modulus was found to significantly increase the $P_{\mathrm{e}}$ value in both cases of isotropic and anisotropic random fields. The increase is greater than one order of magnitude for both cases of isotropic and anisotropic random fields when $\mathrm{COV}_{E}$ increases from $10 \%$ to $15 \%$. This means that careful experimental investigations concerning the variability of this parameter are necessary to lead to reliable results.

\section{References}

Au, S.K. and Beck, J.L., 2001. Estimation of small failure probabilities in high dimensions by subset simulation. Probabilistic Engineering Mechanics, 16, 263-277.

Au, S.K. and Beck, J.L., 2003. Subset simulation and its application to seismic risk based on dynamic analysis. Journal of Engineering Mechanics, ASCE, 129 (8), 901-917.

Au, S.K., Cao, Z.J., and Wang, Y., 2010. Implementing advanced Monte Carlo simulation under spreadsheet environment. Structural Safety, 32 (5), 281-292.

Babu, S.G.L., Srivastava, A., and Murthy, D.S.N., 2006. Reliability analysis of the bearing capacity of a shallow foundation resting on cohesive soil. Canadian Geotechnical Journal, 43, 217-223.

Baecher, G.B. and Christian, J.T., 2003. Reliability and statistics in geotechnical engineering. New York: Wiley.

Cho, S.E., 2010. Probabilistic assessment of slope stability that considers the spatial variability of soil properties. Journal of Geotechnical and Geoenvironmental Engineering, ASCE, 136 (7), 975-984.

Cho, S.E. and Park, H.C., 2010. Effect of spatial variability of cross-correlated soil properties on bearing capacity of strip footing. International Journal for Numerical and Analytical Methods in Geomechanics, 34, 1-26.

Fenton, G.A. and Griffiths, D.V., 2002. Probabilistic foundation settlement on a spatially random soil. Journal of Geotechnical and Geoenvironmental Engineering, ASCE, 128 (5), 381-390.

Fenton, G.A. and Griffiths, D.V., 2003. Bearing capacity prediction of spatially random c-f soils. Canadian Geotechnical Journal, 40 (1), 54-65.

Fenton, G.A. and Griffiths, D.V., 2005. Three-dimensional probabilistic foundation settlement. Journal of Geotechnical and Geoenvironmental Engineering, ASCE, 131 (2), 232-239.

Fenton, G.A., Zhuo, H., Jaksa, M.B., and Griffiths, D.V. 2003. Reliability analysis of a strip footing designed against settlement. In: A. Der Kiureghian, S. Madanat, and J.M. Pestana, eds. Applications of statistics and probability in civil engineering, Millpress, Rotterdam, ISBN: 905966004 8, 1971-1977. 
Griffiths, D.V., Fenton, G.A., and Manoharan, N., 2006. Undrained bearing capacity of two-strip footings on spatially random soil. International Journal of Geomechanics, 6 (6), 421-427.

Honjo, Y., 2008. Monte Carlo simulation in reliability analysis. In: K.K. Phoon, ed. Reliability-based design in geotechnical engineering: computations and applications. Oxford: Taylor \& Francis, 169-171.

Popescu, R., Deodatis, G., and Nobahar, A., 2005. Effect of random heterogeneity of soil properties on bearing capacity. Probabilistic Engineering Mechanics, 20, 324-341.

Santoso, A.M., Phoon, K.K., and Quek, S.T. 2010. Modified Metropolis-Hastings algorithm with reduced chaincorrelation for efficient subset simulation. Probabilistic Engineering Mechanics, 26 (2011), 331-341.
Soubra, A.-H., Youssef Abdel Massih, D., and Kalfa, M. 2008. Bearing capacity of foundations resting on a spatially random soil. Proceedings of GeoCongress 2008, 9-12 March 2008, Orleans, Louisiana: Geotechnical Special Publication No. 178, Sponsored by GEO Institute of ASCE.

Spanos, P.D. and Ghanem, R., 1989. Stochastic finite element expansion for random media. Journal of Engineering Mechanics ASCE, 115 (5), 1035-1053.

Sudret, B. and Berveiller, M., 2008. Stochastic finite element methods in geotechnical engineering. In: K.K. Phoon, ed. Reliability-based design in geotechnical engineering: computations and applications. Oxford: Taylor \& Francis, 260-296. 